\#096 Avaliação in vitro de dois cimentos endodônticos na adesão às paredes do canal radicular

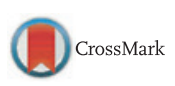

Inês Duarte*, Eunice Virgínia Carrilho, Nuno Ferreira, Francisco Caramelo, Manuel Marques Ferreira

Instituto de Biofísica-IBILI; Faculdade de Medicina da Universidade de Coimbra (FMUC); Instituto de Investigação Clínica e Biomédica de Coimbra da FMUC

Objetivos: Este estudo teve como objetivo avaliar a força de adesão às paredes do canal radicular, de dois cimentos endodônticos, AH-Plus ${ }^{\circledR}$ e GuttaFlow Bioseal ${ }^{\circledR}$.

Materiais e métodos: Para a sua realização foram recolhidos 30 dentes humanos monorradiculares. Após seccionar as coroas dos dentes, estes foram instrumentados mecanicamente com limas ProTaper ${ }^{\circledR}$ Universal ao comprimento de trabalho e divididos em dois grupos. No grupo experimental 1, os dentes foram obturados com AH-Plus ${ }^{\circledR}$, através da técnica de cone único, e no grupo experimental 2, os dentes foram obturados com GuttaFlow Bioseal ${ }^{\circledR}$, também através da técnica de cone único. A avaliação das forças de adesão foi feita através do teste push-out realizado numa máquina universal. O valor da força no momento do deslocamento foi obtido (em Newton) para cada amostra, o que tomando em conta as respetivas áreas de adesão do cimento permitiu o cálculo da força de adesão (em Megapascal).

Resultados: Os resultados demonstram que existem diferenças estatisticamente significativas entre o AH-Plus ${ }^{\circledR}$ e o GuttaFlow Bioseal ${ }^{\circledR}$ nas zonas apical $(p=0,001)$ e média $(p=0,003)$ e que o AH-Plus ${ }^{\circledR}$ proporcionou valores de adesão mais elevados do que o GuttaFlow Bioseal ${ }^{\circledR}$ nas três zonas da raiz do dente.

Conclusões: Existem diferenças significativas na força de adesão entre os dois cimentos, sendo o AH-Plus ${ }^{\circledR}$ o mais eficaz. http://doi.org/10.24873/j.rpemd.2018.11.331

\#097 Eficácia na remoção de smear layer entre dois sistemas de agitação da solução irrigante

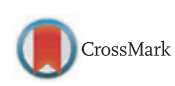

Rita Balaus Custódio*, João Paulo Mouta Dias, Manuel Marques Ferreira

Instituto Pedro Nunes, Faculdade de Medicina da Universidade de Coimbra

Objetivos: A irrigação é um passo crucial para garantir o sucesso dos tratamentos endodônticos e, enquanto que a irrigação convencional é amplamente aceite, novos métodos para melhorar a limpeza canal têm sido desenvolvidos. O propósito deste estudo foi comparar a eficácia na remoção de smear layer entre a técnica convencional de irrigação e dois sistemas de agitação da solução irrigante: um sónico e outro mecânico.

Materiais e métodos: 30 dentes humanos monorradiculares foram recolhidos, cortados a um comprimento de $15 \mathrm{~mm}$ e instrumentados usando o sistema mecanizado ProTaper Universal até um calibre apical F3, sendo irrigados unicamente com hipoclorito de sódio 2,5\%. As amostras foram divididas aleatoriamente em 3 grupos $(n=10)$ e sujeitas a diferentes protocolos de irrigação final: Grupo CI- irrigação convencional; Grupo XPagitação da solução irrigantes com recurso à lima XP-
-Endo Finisher (FKG Dentaire, Suíça); Grupo EDDYagitação da solução irrigante utilizando o sistema sónico EDDY (VDW, Alemanha). As raízes foram cortadas longitudinalmente e examinadas ao microscópio eletrónico de varrimento (MEV) com uma magnificação de $1000 \mathrm{x}$, obtendo microfotografias de todos os terços canalares para cada amostra. A presença de smear layer foi avaliada a 2, 8 e 12 mm de distância ao apéx de acordo com uma escala de 5 graus. Os dados resultantes foram analisados estatisticamente recorrendo ao teste ANOVA dois fatores.

Resultados: O grupo de irrigação convencional apresentou um valor médio superior de smear layer, mas a diferença entre os grupos experimentais não foi estatisticamente significativa ( $p=0,641)$. O valor médio de smear layer foi maior para o terço apical e menor para o terço médio, sendo esta discrepância estatisticamente significativa $(p<0,05)$.

Conclusões: Quando utilizado apenas hipoclorito de sódio 2,5\% como solução irrigante, não se verificaram diferenças estatisticamente significativas na limpeza canalar entre a irrigação convencional e os métodos de agitação analisados, embora estes tenham demonstrado valores médios de smear layer mais baixos. http://doi.org/10.24873/j.rpemd.2018.11.332

\#098 Nova proposta de associação de solventes para o retratamento endodôntico - estudo in vitro

Inês Ribeiro Valente Lucas Ferreira*, Ana Cristina Braga, Maria Ascensão Lopes, Irene Pina-Vaz

Faculdade de Medicina Dentária da Universidade do Porto; Faculdade de Medicina Universidade Porto; REQUIMTE-LAQV; Departamento de Engenharia Metalúrgica e de Materiais, Faculdade de Engenharia da Universidade do Porto; Departamento de Produção e Sistemas da Escola de Engenharia, Centro ALGORITMI, Universidade do Minho

Objetivos: Avaliar a eficácia da associação de solventes na dissolução da guta-percha e cimento endodôntico à base de resina epóxi.

Materiais e métodos: Moldes de aço inoxidável $(7 \mathrm{~mm}$ x 3 $\mathrm{mm}$ ) foram preparados e preenchidos com guta-percha (Dentsply Maillefer) e cimento AH Plus ${ }^{\circledR}$ (Dentsply DeTrey, Konstanz, Germany), separadamente. As amostras $(n=15)$ foram expostas a 3 associações de solventes: Metiletilcetona Tetracloroetileno (1:1); Metiletilcetona Óleo de laranja (1:1); Metiletilcetona Eucaliptol (1:1) e submetidas à agitação por ultrassom durante 2 e 5 minutos. A dissolução da guta-percha e do cimento AH Plus nas diferentes associações de solventes foi obtida pela diferença de peso pré e pós imersão (adaptação da norma ISO 6876:2012). Os resultados obtidos foram submetidos à análise de variância (ANOVA), considerando um nível de significância de 0,05.

Resultados: Relativamente à dissolução da guta-percha, detetaram-se diferenças estatisticamente significativas entre as 3 associações $(p<0,05)$. O tempo não foi significativo. As amostra submetidas à associação Metiletilcetona Tetracloroetileno, apresentaram a maior percentagem de perda de peso. Em relação ao cimento AH Plus, não houve diferenças estatisticamente significativas entre as associações: Metiletilcetona Tetracloroetileno e Metiletilcetona Óleo de laranja $(p=0,304)$. A dissolução do cimento foi diretamente proporcional ao tempo de imersão. A 
associação Metiletilcetona Eucaliptol revelou ser a associação menos eficaz, tanto para a guta-percha como para o cimento.

Conclusões: As duas associações Metiletilcetona Tetracloroetileno e Metiletilcetona Óleo de laranja apresentaram uma eficácia de dissolução semelhante na guta-percha e cimento, superior à associação Metiletilcetona Eucaliptol. A influência do tempo foi mais evidente na dissolução do cimento. A associação de solventes com especificidade para diferentes materiais obturadores, poderá, num só passo, aumentar a eliminação de remanescentes potencialmente infetados, melhorando o prognóstico do retratamento endodôntico.

http://doi.org/10.24873/j.rpemd.2018.11.333

\section{\#099 Tendências da Medicação Intracanalar entre os Membros da Sociedade Portuguesa de Endodonto}

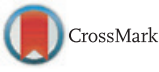

Mariana Peixe Domingos Alves Pires*,

João Ferreira Meirinhos, Joana Sofia Cordeiro Martins, Mário Rito Pereira, António Ginjeira

Faculdade de Medicina Dentária, Universidade de Lisboa

Objetivos: O objetivo deste estudo foi determinar as tendências atuais da utilização de medicação intracanalar em tratamentos endodônticos numa população específica em Portugal.

Materiais e métodos: Foi enviado, via correio eletrónico, um convite para responder a um questionário online a 134 membros da Sociedade Portuguesa de Endodontologia. O tema central abordado no questionário foi a seleção da medicação intracanalar: qual a medicação que utilizam, se utilizam, e em que situações o fazem.

Resultados: Foram obtidas um total de 48 respostas. Os dados demonstram que mais de $58 \%$ dos inquiridos inclui no seu protocolo clínico a utilização de medicação intracanalar. O hidróxido de cálcio foi considerado o material de eleição, sendo que 10 respostas indicam a sua aplicação em $100 \%$ dos casos. $60 \%$ dos participantes mencionaram que a escolha e uso da medicação intracanalar depende do diagnóstico pulpar e/ ou periapical. No entanto, mais de $53 \%$ dos intervenientes reportaram que não consideram o uso de medicação intracanalar fundamental para o sucesso do tratamento endodôntico.

Conclusões: A maioria dos participantes utiliza medicação intracanalar na sua prática clínica, no entanto, a maioria reitera que a sua aplicação não é fundamental para o sucesso do tratamento endodôntico.

http://doi.org/10.24873/j.rpemd.2018.11.334

\#101 Estudo de caso-controlo dos impactos na auto-percepção da saúde oral em diabéticos tipo 2

José Frias-Bulhosa*, Maria Conceição Manso, Carla Lopes da Mota, Paulo Melo

USF Barão do Corvo, ACES Gaia., FCS-UFP; (FP-ENAS), LAQV/REQUIMTE da Univ. do Porto, FCS-UFP; Departamento de Saúde Pública Oral-Instituto de Saúde Pública da Universidade do Porto, FMDUP; EPIUnit

Objetivos: A saúde é um conceito multidimensional que na actualidade implica uma combinação entre a auto- -percepção da saúde por parte dos indivíduos e a avaliação clínica realizada pelos profissionais de saúde. Esta combinação caracteriza-se por resultar da associação entre conceitos objectivos e subjectivos que exprimem-se de forma dinâmica ao longo do tempo de vida dos indivíduos e em função das condições de saúde vivenciadas no passado e no presente. Este estudo tem como objetivo verificar se há diferenças entre a auto-avaliação da saúde oral entre pacientes com diabetes mellitus tipo 2 (DM2) e sem diabetes (nDM2) e explorar as condições de saúde geral e oral, associadas à avaliação da auto-percepção de impactos na saúde oral.

Materiais e métodos: Após aprovação da Comissão de Ética da ARS-N estabeleceu-se uma amostra aleatória simples, com base na população inscrita com DM2 na USF de Espinho para recolha de consentimento informado e participação voluntária de 343 adultos com DM2 e de 323 nDM2. Os dados de clínica geral e de saúde oral (índices: CPOd e o Periodontal Comunitário) foram coletados segundo critérios da DGS e da OMS e realizada pergunta para auto-avaliação de saúde oral numa escala de Likert (5 pontos). A análise inferencial utilizou testes não-paramétricos e regressão logística multivariada (RLM) (alfa=0,05).

Resultados: A maioria dos participantes foi do género feminino (56,9\%), a média de idade da amostra foi de $67.8 \pm 9.8$ (DM2) e de 59.8 \pm 14.2 (nDM2) anos. Os pacientes DM2 autoavaliam significativamente pior a sua saúde oral do que os nDM2 $(\mathrm{p}<0,001)$, avaliando mais frequentemente e de forma significativa $(p<0,001)$ as perceções de 'má' ou 'muito má'. Nos nDM2, a RLM mostrou que a presença de >= 20 dentes é um factor de protecção para auto-percepção da saúde oral como 'mediana', 'boa' ou 'muito boa'; já a presença de cálculo ( $O R=3,55, p=0,049)$ ou bolsas periodontais $(\mathrm{OR}=4,32, \mathrm{p}=0,025)$ são fatores de risco para uma perceção de pior saúde oral; entre os DM2 é a duração da diabetes, consumo de tabaco, ter pelo menos um dente perdido, pelo menos um dente obturado, CPOd > 0, uso de prótese removível ou não reabilitação são factores de risco para auto-avaliação como 'muito má' ou 'má' saúde oral e o número de dentes >= 20 como factor proctetor.

Conclusões: Este estudo confirma a hipótese que existe diferenças na auto-percepção da sua saúde oral entre os DM2 e nDM2. Indivíduos com DM2 mostraram uma pior auto-percepção de saúde oral do que os nDM2, classificando-a mais frequentemente como 'má ou 'muito má'.

http://doi.org/10.24873/j.rpemd.2018.11.335

\#102 Ausência por extracção do primeiro molar definitivo nos paciente da CDEM

António Tenreiro Lopes*, Pedro Silva Rodrigues, Cátia Sofia Carriço Simões, Eduardo Manuel Soares Guerreiro, Irina Xavier, Tiago Filipe Rodrigues Dionísio

Instituto Superior de Ciências da Saúde Egas Moniz

Objetivos: A cárie dentária é a causa mais comum da exodontia dos primeiros molares definitivos, sendo o primeiro molar inferior o mais afectado. O objectivo do nosso trabalho é estudar a prevalência da ausência por extracção do primeiro molar definitivo nos paciente jovens, dos 16 aos 30 anos, observados na consulta de triagem da Clínica Dentária Egas Moniz. 